\title{
Nanocrystalline Materials: Interfaces and Mesoscopic Correlations Studied by Neutron Scattering
}

\author{
U. Stuhr*, W. WAGNer, J. KohlBRECher \\ AND H. VAN SWYGENHOVEN \\ Paul-Scherrer-Institut, 5232 Villigen PSI, Switzerland
}

\begin{abstract}
Nanocrystalline materials can exhibit properties which are considerably different from their coarse-grained counterparts, making them unique for basic or applied research and also very promising for potential applications. The topics which are addressed in the present paper are magnetic properties and magnetic correlations on the nanometer scale, mechanical properties, in particular the influence of grain boundaries on the elastic and plastic behaviour, and vibration modes of grain boundary atoms in nanostructured materials.
\end{abstract}

PACS numbers: $61.46 .+\mathrm{w}, 63.22 .+\mathrm{m}, 75.20$.En, $75.50 . \mathrm{Tt}$

\section{Introduction}

Nanocrystalline materials are polycrystals - consisting of quite conventional substances like metals, alloys or ceramics - with the specific feature that the grain size is of the order of a few to some tens of nanometers. The overall properties of that type of material, such as electrical, mechanical and magnetic are to a great extent determined by the presence and the nature of the large amount of grain boundaries or interfaces. As a second aspect, the grain size frequently is in the range of or even beyond the critical length scale for physical properties, like domain wall width in magnetism, free path length in electronic motion etc., giving these materials the character of mesoscopic systems. As a consequence, the properties of these materials can be significantly altered compared to their coarse grained counterparts, making them interesting for potential novel applications in technology as well as in the field of basic science. For the latter, the high volume fraction

* corresponding author; e-mail: uwe.stuhr@ psi.ch 
of interfaces and the mesoscopic length scale allows to study specific phenomena where the influence of the one or the other dominates the properties.

From the mid-80's, increasing interest in these materials can be recognized, stimulated by Gleiter's initiative for a controlled laboratory synthesis by the inert-gas condensation (IGC) technique [1]. Since then, numerous publications and conference proceedings $[2,3]$ document the relevance meanwhile attributed to nanocrystalline materials. In this period not only the interest has increased but also the objectives have changed: from general interest in novel properties at the beginning over well-characterized synthesis and systematic studies of selected topics towards emphasis for applications in industry, environment, and technology. Along with this development, also the methods for synthesis and processing were broadened and refined following different chemical or physical routes, not at least aiming at large-scale production for technological application.

The present article presents selected topics related to nanocrystalline materials based on pure metals or alloys, synthesized primarily by the IGC technique, but also by thermodynamically driven phase separation [4] or, in computer simulations, by filling an assigned volume with nanocrystalline grains nucleating from stochastically chosen seeds [5]. The general objective in most cases is to relate macroscopic, phenomenological properties to structure and physical phenomena on the nanometer scale. Hence, for the experimental characterization, when insight into the material with nanometer scale resolution is required, neutron- or $\mathrm{X}$-ray (synchrotron) scattering often plays a key role, as will be demonstrated in the following. In complement to the experimental investigations, molecular dynamics computer simulations give valuable additional input. All together combines to a set of tools which should allow a conclusive interpretation and understanding of the microstructure-properties relationship.

\section{Structure and properties}

\subsection{Magnetic properties of nanostructured Fe and Co}

The particular morphology of nanocrystalline materials has multiple influences on the magnetic properties. Whereas isolated magnetic clusters above the onset of superparamagnetism are magnetically hard [6], nanostructured magnets consisting of consolidated grains often have superior soft magnetic properties [7]. Remarkably, the coercivity in such materials can be tuned by a variation of the grain size. This was found for instance in nanocrystalline Fe where the coercivity shows a distinct maximum at grain sizes of about $35 \mathrm{~nm}$, falling off sharply towards smaller grains [8].

It is evident that the interplay of structure and magnetic correlations on the nanoscale play a key role for the explanation of this behavior. Magnetic small angle neutron scattering (SANS) is the only technique which directly can analyze magnetic correlations on the nanoscale. With or without an external field applied, 
one can distinguish the spontaneous magnetic correlations and their response to an external field. Figure 1 shows examples of lines of equal intensity (two-dimensional isointensity contours) from a nanocrystalline Fe sample, superposing nuclear scattering and magnetic scattering

$$
\frac{\mathrm{d} \sigma}{\mathrm{d} \Omega}(\boldsymbol{Q})=A(Q)+B(Q) \sin ^{2} \psi,
$$

where $\psi$ is the azimuthal angle between the direction of the local magnetization $\boldsymbol{M}$ and the scattering vector $\boldsymbol{Q}$, and $A(Q)$ and $B(Q)$ are the nuclear and magnetic scattering terms, respectively

$$
\begin{aligned}
& A(Q)=(\Delta b)^{2}\left|F_{\mathrm{N}}(Q, R)\right|^{2}, \\
& B(Q)=(\Delta p)^{2}\left|F_{\mathrm{M}}(Q, R)\right|^{2},
\end{aligned}
$$

with $(\Delta b)^{2}$ and $(\Delta p)^{2}$ - the contrast factors, and $F_{\mathrm{N}}, F_{\mathrm{M}}$ - the structure functions for nuclear and magnetic scattering, respectively. In zero field, the magnetic scattering, too, averages isotropically (see Fig. 1), due to the arbitrarily directing individual magnetic moments, whereas in the (saturating) field of $10 \mathrm{kOe}$ the $\sin ^{2} \Psi$-dependence can clearly be seen.
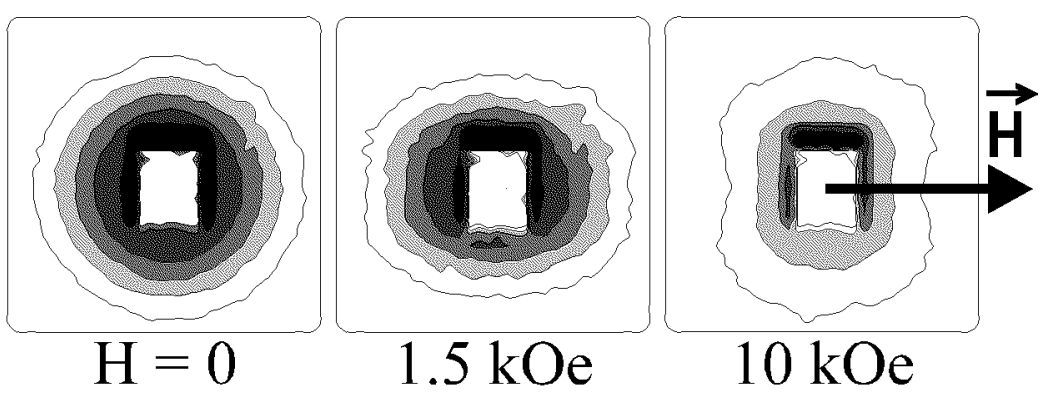

Fig. 1. SANS isointensity contours on a two-dimensional detector, for a nanocrystalline Fe-sample measured in zero field and in external magnetic fields as indicated (the $Q$-range covered extends to about $0.16 \mathrm{~nm}^{-1}$ ).

Averaging both pattern with regard to the azimuthal angle around the central beam results in the radial scattering cross-section shown in Fig. 2, along with another equivalent example for a nanocrystalline Co sample.

The scattering curves measured with and without magnetic field lie close together and are parallel for large $Q$-values; for smaller $Q$ a significant splitting is observed, which is due to extra magnetic scattering in zero field. This scattering gives direct evidence for extended magnetic correlations exceeding the grain size, thus confining many grains to a correlated magnetic alignment. A quantitative analysis of the SANS data $[9,10]$ from samples of different grain sizes results in the plot of Fig. 3 where the magnetic correlation length of nanocrystalline Fe is 

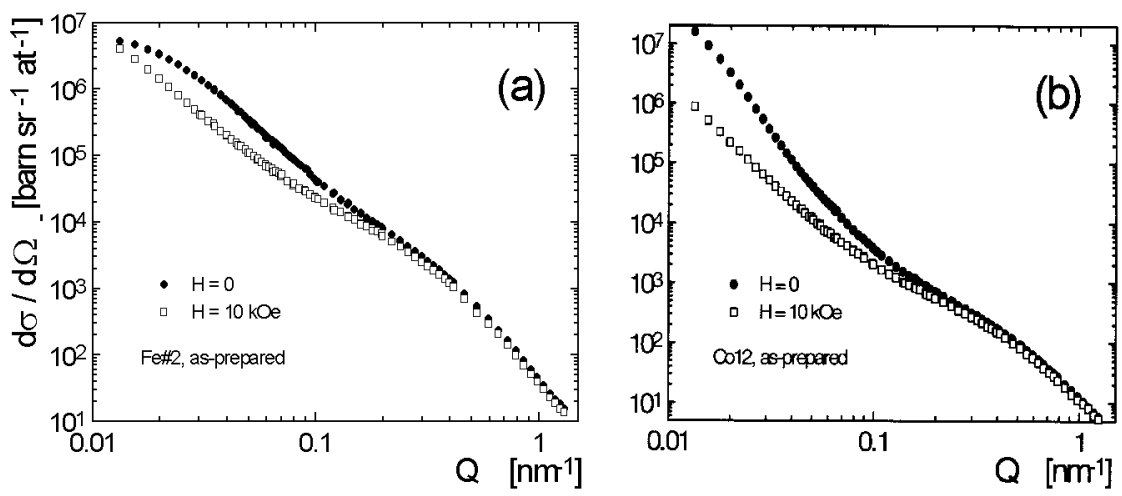

Fig. 2. Radial scattering cross-section for as-prepared nanostructured Fe (a) and Co (b) measured at $H=0$ and $H=10 \mathrm{kOe}$

shown as a function of grain size. Shortest correlations are found for grain sizes around 25 to $30 \mathrm{~nm}$ which is the range of the bulk domain-wall width. These results are quantitatively understood and explained within the frame of the random anisotropy model (RAM) $[11,12]$, generalized to take into account domain-wall formation within grains and reduced interface coupling [13]. It explains quantitatively the sudden and strong magnetic softening occurring below the grain size of minimum correlation.

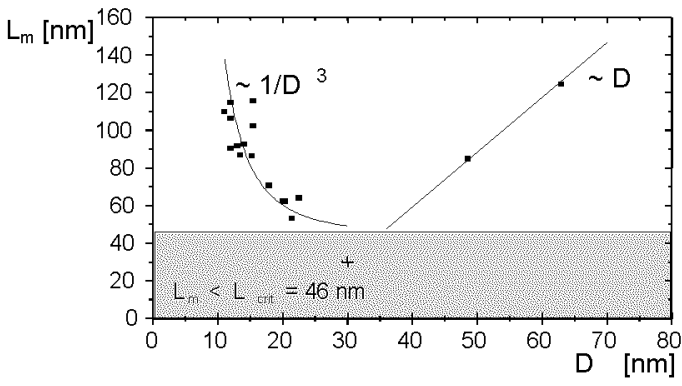

Fig. 3. Average spatial magnetic correlation length $L_{\mathrm{m}}$ versus average grain size $D$, for nanostructured Fe. The solid line in the low grain size regime is primarily a guide to the eye, but also represents a fit proportional to $1 / D^{3}$ predicted by the RAM. No magnetic correlations with $L_{\mathrm{m}}<L_{c r i t}$ are found (gray shadowed region).

In Fig. 1, at the intermediate field of $1.5 \mathrm{kOe}$, the intensity contours are found elongated parallel to the external field, which reflects a net magnetic component of the correlated grains directing perpendicular to the field. This effect, observed also in other samples of different material (e.g. Co), different grain size and at different fields, reflects that the domains, confining several grains, remain tilted 
considerably out of the field direction even in fields up to several kOe. Although this behaviour seems energetically unfavourable at a first glance, the effect and its dynamics can well be described by a model which minimizes the configuration energy of a magnetically anisotropic cluster in an external field, the Stoner-Wohlfarth model [14], here modified to account for the magnetic exchange between the grains. Calculating the two-dimensional magnetic scattering of the related modeled microstructure (tilted ferromagnetic domains in a homogeneous magnetic matrix) results in the observed intensity contours when the tilting angles lie in a cone of up to $70^{\circ}$ opening angle [15].

\subsection{Magnetic nanostructure in Fe-Si-B-based alloys}

Whereas the samples treated in the previous section were produced by the IGC-technique, the route of synthesis followed here starts from metallic glasses which undergo a decomposition and nanocrystallization upon annealing at temperatures near their glass transition temperature $T_{\mathrm{g}}$. Our example refers to nanocrystalline materials of the FeSiBNbCu system, well known for their soft magnetic properties, i.e. high permeability, low coercivity and low magnetostriction $[16,17]$. They are prepared from the melt spun amorphous alloy by thermal treatment. Annealing at $823 \mathrm{~K}$ for $1 \mathrm{~h}$ produces nanosized $\alpha-\mathrm{Fe}(\mathrm{Si})$ crystallites of about $5 \mathrm{~nm}$ in radius embedded in an amorphous matrix. The excellent soft magnetic properties of these materials have attracted much attention for structural and magnetic studies. It is known that the combined addition of copper and niobium to the amorphous $\mathrm{Fe}-\mathrm{Si}-\mathrm{B}$ alloy is crucial for the formation of the nanostructured morphology. The addition of $\mathrm{Cu}$ enhances the nucleation of $\alpha-\mathrm{Fe}(\mathrm{Si})$ grains, while the slow-diffusing $\mathrm{Nb}$ hinders a rapid growth.

The low coercivity of these alloys in the literature is frequently attributed to random anisotropy effects $[16,18]$. As outlined in the previous section, one of the basic assumptions for applying the RAM is the presence of a magnetic exchange coupling across the interface, here across the amorphous-crystalline phase boundary. Again, the SANS technique is used to characterize the magnetic microstructure and correlations in nanocrystalline FeSiBNbCu. Field dependent SANS measurements have been performed in different temperature regimes related to crystallization temperature $\left(T_{\mathrm{x}}=750 \mathrm{~K}\right)$ and Curie temperatures of the nanocrystalline precipitates $\left(T_{\mathrm{C}}^{\mathrm{cr}} \approx 900 \mathrm{~K}\right)$ and of the amorphous phase $\left(T_{\mathrm{C}}^{\mathrm{am}}\right)$, the latter being lower than the crystallization temperature $T_{\mathrm{x}}$. Hence, at room temperature the material shows ferromagnetic behaviours for both phases. With increasing temperature the saturation magnetization of the two phases is reduced until the amorphous phase becomes paramagnetic at $T=T_{c}^{\mathrm{am}}$. At still higher temperatures $\left(T_{\mathrm{c}}^{\mathrm{am}}<T<T_{\mathrm{c}}^{\mathrm{cr}}\right)$ we observe ferromagnetic single-domain crystals showing superparamagnetic properties.

For a quantitative analysis of the temperature and field dependent scattering of our alloy, again Eq. (2.1) applies, however, with different definitions for 
the coefficients $A(Q)$ and $B(Q)$ in order to account for the specific conditions of the two-phase alloy. We consider ferromagnetic single domain precipitates with magnetization $M_{\mathrm{s}}^{\mathrm{cr}}$ in a ferromagnetic amorphous matrix $M_{\mathrm{s}}^{\mathrm{am}}$.

Orientation and amplitude of the magnetization in the two phases may be different. Again, there are two scattering contributions of the precipitates: the nuclear scattering $F_{\mathrm{N}}^{2}(\mathrm{Q})$ and the magnetic scattering $\tilde{F}_{\mathrm{M}}^{2}(\mathrm{Q})$, the latter measuring fluctuations in both, amplitude and orientation, of the local magnetization. The two coefficients of Eq. (2.1) then become

$$
\begin{aligned}
& A(Q)=F_{\mathrm{N}}^{2}(Q)+\tilde{F}_{\mathrm{M}}^{2}(Q) 2 \frac{L(\alpha)}{\alpha}, \\
& B(Q)=\tilde{F}_{\mathrm{M}}^{2}(Q)\left\{[L(\alpha)-\gamma]^{2}+\hat{L}(\alpha)\right\},
\end{aligned}
$$

where $L(\alpha)=\operatorname{cotan} \alpha-1 / \alpha$ is the Langevin function, $\alpha=\mu_{0}\left(H+M_{\mathrm{eff}}\right) M_{\mathrm{s}}^{\mathrm{cr}} V_{\mathrm{P}} / k T$, $\gamma=M_{\mathrm{s}}^{\mathrm{am}} / M_{\mathrm{s}}^{\mathrm{cr}}, \hat{L}(\alpha)=1-L^{2}(\alpha)-3 L(\alpha) / \alpha$ and $\tilde{F}_{\mathrm{M}}^{2}(Q)=M_{\mathrm{s}}^{\mathrm{cr}} V_{\mathrm{P}} f(Q)$. The terms with $\tilde{F}_{\mathrm{M}}^{2}(Q)$ in Eq. (2.4) describe the transition from anisotropic magnetic scattering for large values of $\alpha$, i.e. high fields and low temperatures, when the alignment is perfect and the isotropic magnetic contribution $\tilde{F}_{\mathrm{M}}^{2}(Q) 2 L(\alpha) / \alpha$ in $A(Q)$ vanishes, to isotropic scattering when the values of $\alpha$ becomes small, i.e. for small magnetic fields and/or higher temperatures. Then the anisotropic magnetic term $B(Q)$ vanishes and the isotropic term approaches $F_{\mathrm{N}}^{2}(Q)+(2 / 3) \tilde{F}_{\mathrm{M}}^{2}(Q)$.

Figure 4 shows the results of the SANS measurements by means of the integrated intensities $\int A(Q) \mathrm{d} Q$ and $\int B(Q) \mathrm{d} Q$. The solid lines are the result of a fit of Eq. (2.4) to the data.
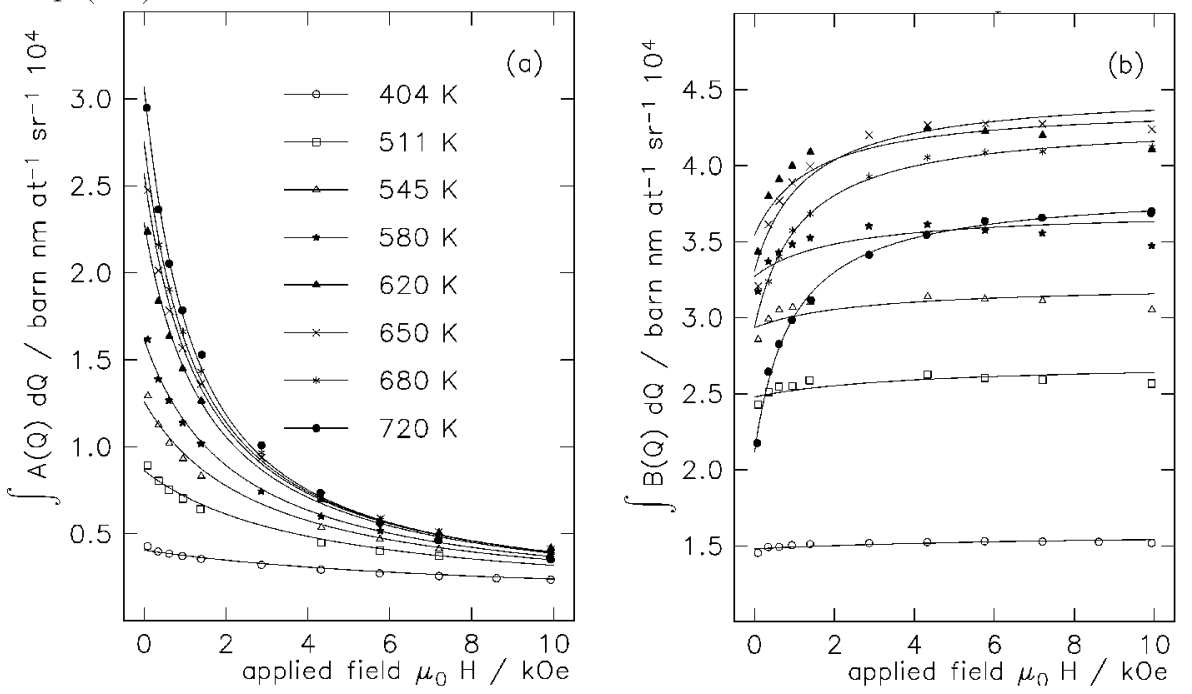

Fig. 4. The integral intensities $\int A(Q) \mathrm{d} Q$ in (a) and $\int B(Q) \mathrm{d} Q$ in (b) follow the field and temperature dependence described by Eq. (2.4). 
The temperature dependent fit parameters $M_{\mathrm{eff}}(T)$ and $\gamma(T)$ are shown in Fig. 5. For $\mu_{0} M_{\text {eff }}(T)$ the fit always yields values below $10 \mathrm{kOe}$. In the case of ferromagnetic exchange coupling between matrix and precipitates one would expect values of about $400-500 \mathrm{kOe}$ for $\mu_{0} M_{\mathrm{eff}}(T)[19,20]$. The much lower magnitude of $\mu_{0} M_{\text {eff }}(T)$ derived from SANS implies that the ferromagnetic single domain $\alpha$-Fe(Si) nanocrystals are coupled by magnetic dipole-dipole interaction to the moments of the ferromagnetic amorphous matrix rather than by (the much stronger) exchange coupling. Hence, in this temperature range the random anisotropy model $[11,12]$, although frequently propagated, cannot be applied to explain the soft magnetic properties of such materials.

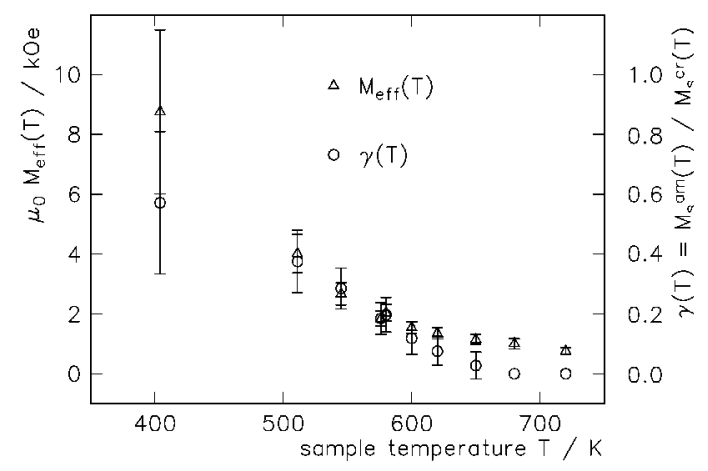

Fig. 5. Effective magnetization $M_{\text {eff }}$ and magnetization ratio $\gamma(T)=M_{\mathrm{s}}^{\mathrm{am}}(T) / M_{\mathrm{s}}^{\mathrm{cr}}(T)$ resulting from a fit of Eqs. (2.4) to the experimental data. The error bars describe the $68.3 \%$ confidence interval.

The unexpected breakdown of exchange interaction between the two ferromagnetic phases suggests the presence of a non-magnetic or paramagnetic interface between the nanocrystals and the matrix. In fact, in situ small-angle X-ray scattering (SAXS) measurements as well as recent SANS measurements using polarized neutrons [21] give clear evidence for a concentration pile-up of $\mathrm{Nb}$ around the $\alpha$ - $\mathrm{Fe}(\mathrm{Si})$ crystals during the (Nb-diffusion controlled) growth of the nanocrystals. The Nb-concentration has a strong influence on the Curie temperature of the amorphous phase: $40 \mathrm{~K}$ decrease for only 1 at.\% increase in Nb concentration [21]. In Fig. 6 the $\mathrm{Nb}$ concentration profile $\left(c_{\mathrm{Nb}}\right)$ is shown as a function of the distance $x$ from the centre of the $\mathrm{Fe}_{3} \mathrm{Si}$ precipitates, together with the local change in Curie temperature, calculated via $T_{\mathrm{c}}(x)=650 \mathrm{~K}-\left\{40 \mathrm{~K}\left[c_{\mathrm{Nb}}(x)-c_{\mathrm{M}}\right]\right\}$. In conclusion, we find that the combination of the low diffusivity of $\mathrm{Nb}$ and the reduction in Curie temperature results in a thin niobium enriched layer at the interface which at intermediate temperatures becomes paramagnetic and prevents exchange coupling between ferromagnetic matrix and ferromagnetic precipitates. 


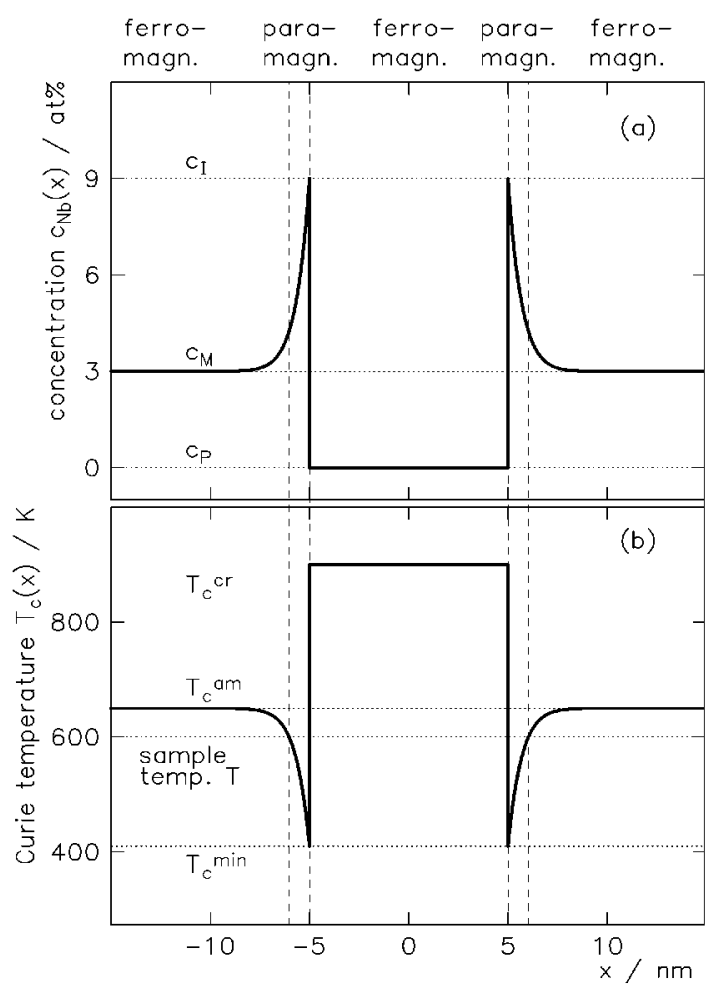

Fig. 6. The top part (a) shows the niobium concentration profile crossing the interface between the matrix and a nanocrystalline precipitate. The horizontal dotted lines indicate the $\mathrm{Nb}$ concentration of the precipitate $\left(c_{\mathrm{p}}\right)$, the matrix $\left(c_{\mathrm{M}}\right)$, and at the interface $\left(c_{I}\right)$. In the lower part (b) the influence of the variation of concentration on the local Curie temperature $T_{c}(x)$ is presented. The minimum local Curie temperature, the Curie temperature of the amorphous and of the crystalline phases are indicated by $T_{\mathrm{c}}^{\mathrm{min}}, T_{\mathrm{c}}^{\mathrm{am}}$ and $T_{c}^{\mathrm{cr}}$, respectively. For temperatures $T_{c}^{\mathrm{min}}<T<T_{c}^{\max }$ both, matrix and precipitates, are ferromagnetic but separated by a thin paramagnetic layer.

\subsection{Interfacial structure and mechanical properties}

The reduction of the grain size down to the nm regime has led to new interesting mechanical properties of metals and alloys. Two open questions are of primary interest: the first addresses the structure of the grain boundaries, whether they are "normal" or unusual, a topic on which quite controversial results have been reported in literature $[22,23]$. The second one addresses the influence of the grain boundaries and grain size on the mechanism for plastic deformation where the relation between yield stress and grain size has been found to follow a quite complex behaviour in nanophase materials [24].

Massive parallel computers offer nowadays the possibility to perform atomic scale molecular dynamics simulations of nanocrystalline metals. We simulated 
three-dimensional nanocrystalline $\mathrm{Ni}$ and $\mathrm{Cu}$ fcc metals in the grain size range 5-12 nm to study the structural and mechanical properties as function of grain size and texture, focusing on the study of the interfaces responsible for the plastic deformation $[23,25-27]$. The comparison of samples which differ only in grain size allows us to study the same grain boundary for different grain sizes, isolating the effect of grain size on grain boundary structure. As an analysis tool, the local crystalline order of each atom in the sample is calculated [28]. This allows the identification of fcc (inside the grains), hcp and other types of defected atoms in the sample. The presence of hcp atoms is relevant because they represent the stacking faults in an fcc structure and hence help visualizing dislocation activity.

Investigating the structure of the grain boundaries on atomic level, we found that grain boundaries in nanophase metals are essentially similar to those found at the microscale, i.e. similar structural units are found, providing evidence against the view of grain boundaries in nanocrystals as amorphous or liquid-like interfaces. Figure 7 gives two examples of grain boundary structures in a $12 \mathrm{~nm}$ Ni sample. Figure 7 a shows a boundary with a misfit of about 5 degrees from the perfect twin. A repeated building structure was found consisting of structural units of $\Sigma=3$ symmetrical tilt twin boundary (black atoms) and highly disordered steps between those structural units (dark-grey atoms). This twin-and-step block is repeated several times forming a stair with the twins in parallel but non-consecutive (111) planes.

The corresponding grain boundary in the $5.2 \mathrm{~nm}$ sample shows a similar structural unit [23], in this case, however, there is no clear repetition of this unit due to the restriction imposed by the smaller grain size. This shows that the structural features of the boundary are basically independent of grain size in this range.

Figure $7 \mathrm{~b}$ shows another grain boundary in the $\mathrm{Ni} 12 \mathrm{~nm}$ sample with a misfit of about 50 degrees between the [100] directions. The misorientation is not close to any special boundary but rather arbitrary. The grain boundary is not amorphous, and a significant degree of structural coherence across the grain boundary plane can be observed between the crystallographic planes. The misfit due to the different interplanar distance for the different types of planes is accommodated by more disordered region every three planes [23].

The computer simulations provide also access to a microscopic view of the deformation processes. One of the most striking features is the observation of a change in plastic regime as the grain size approaches the $\approx 10 \mathrm{~nm}$ region. In fact, intragrain dislocation activity ceases to operate below this size and grain boundary sliding, a process based on mechanical and thermally activated single atomic jumps, dominates the contribution to deformation. It in turn implies that deformation becomes easier as the grain size further decreases.

X-ray diffraction peak profile analysis has been successfully used in the analysis of the densities and arrangements of dislocations as well as the resid- 
(a)

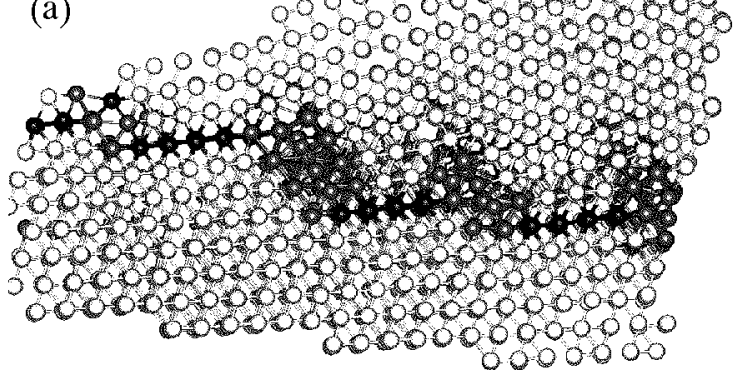

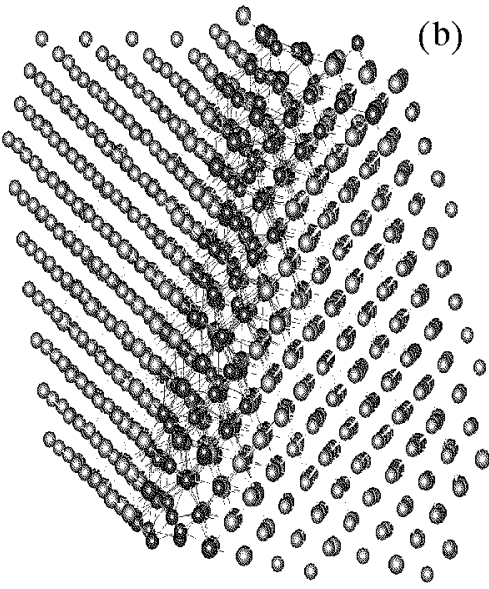

Fig. 7. Two grain boundaries in a $12 \mathrm{~nm}$ sample. (a) View perpendicular to the grain boundary plane, along a common axis, which is close to a $\langle 110\rangle$ direction. The misorientation between the grains is 75 degrees; (b) grain boundary with a misorientation of 50 degrees. (For the present black and white figures we use light-grey for the fcc atoms, black for hcp atoms and dark-grey for all types of non-fcc atoms. In [23, 25-27] different colours are used for each type of atoms, which makes the pictures more clear.).

ual long-range internal stresses [29]. Since the reflected intensity of a dislocation depends on the orientation of its Burgers vector relative to the diffraction vector, it is also possible to evaluate the dominant Burgers vector of dislocations with the diffraction technique. First results on the presence of dislocations in nanocrystalline $\mathrm{Cu}$ have demonstrated the usefulness of this technique. Even when a rotating anode is used, the footprint of the X-ray beam at the sample has to be kept at a few tenths of $\mathrm{mm}$ in size because of intensity reasons. Such focus is suitable for a macroscopic investigation of dislocation arrangements, since it integrates over many grains of the sample. Synchrotron radiation sources reduce the footprint of the X-ray beam on the specimen down to a few tens of micrometer, allowing the investigation of microscopic dislocation arrangements. Experiments to investigate the intrinsic grain boundary dislocations and the dislocations formed during plastic deformation in nanocrystalline metals are planned at the Swiss Light Source.

\subsection{Local vibrational density of states in nanocrystalline Pd}

A quite different approach to characterize the grain boundaries of nanocrystalline materials makes use of the dynamics of the atoms in this region. The vibrations of the grain boundary atoms are expected to be quite different compared to inner-grain atoms since interfaces are essentially two-dimensional and, at least in some nanocrystalline materials, the atomic structure may have a high degree of disorder. Earlier investigations of the dynamics by specific heat, neutron spec- 
troscopy and resonant $\gamma$-spectroscopy $[30,31]$ showed that the low frequency vibrational densities of states (VDOS) of nanocrystalline materials are enhanced compared to ordinary poly- or single-crystalline materials. However, these experiments could not distinguish between interface (grain boundaries or inner surfaces) and inner-grain regions. The aim of a recent study [32] was to investigate the (local) VDOS of the interfaces and the inner-grain regions in nanocrystalline $\mathrm{Pd}$ separately.

The VDOS can best and most directly be observed by incoherent inelastic neutron scattering. For a separate investigation of the vibrations of the interface atoms it is necessary to enhance the contrast between the inner-grain regions and the grain boundaries. Hydrogen is an ideal probe for the investigation of interface vibrations since (i) it is a strong incoherent scatterer, (ii) it preferably occupies sites at interfaces $[33,34]$ and, (iii) at low frequencies, the light hydrogen atoms follow the vibrations of the neighbouring (heavier) metal atoms [35] (the extra hydrogen modes occur at energies above $50 \mathrm{meV}$ [33]). Therefore, the low frequency neutron spectrum of the hydrogen atoms reflects essentially the vibrational spectrum of the metal atoms at the interfaces. The experiment was performed with nanocrystalline $\mathrm{Pd}$ because $\mathrm{Pd}$ has a nearly pure coherent scattering cross-section, it can be doped with hydrogen at room temperature, and it is well established that the hydrogen atoms are located in the grain boundaries. The neutron spin-flip probability for (spin-) incoherent and coherent scattering is $2 / 3$ and 0 , respectively. Therefore the incoherent scattering of the hydrogen can be separated from the coherent Pd scattering by neutron polarization analysis.

The experiment was performed at the time-of-flight spectrometer D7 at the Institut Laue-Langevin in Grenoble which is equipped with supermirror polarizers and analyzers. The nanocrystalline $\mathrm{Pd}$ sample was prepared by the inert gas condensation method and doped with 2.9 at\% hydrogen at 3 mbar gas pressure. The coherent and incoherent scattering intensities of the nanocrystalline $\mathrm{P} d \mathrm{H}_{0.03}$ sample for two different temperatures are given in Figs. 8a and b, respectively. The data were treated as follows: (i) calculation of coherent and incoherent spectra from the neutron spin-flip and non-spin-flip data, (ii) subtraction of the inelastic background as determined from spectra taken at $2 \mathrm{~K}$ and (iii) dividing the intensities by the transmission of the polarization-analyzers and the detector efficiency.

The incoherent scattering law $S(Q, \omega)$ can be written in the one-phonon approximation as

$$
S(\boldsymbol{Q}, \omega)=\mathrm{e}^{-2 W}\left[\delta(\omega)+f(\omega) \frac{\boldsymbol{Q}^{2} k_{\mathrm{B}} T}{2 M \omega^{2}}\right]
$$

where $\hbar Q$ and $\hbar \omega$ are the neutron momentum and energy transfers, respectively ( $h$ is Planck's constant), $W$ is the Debye-Waller exponent, $k_{\mathrm{B}}$ is Boltzmann's constant, and $M$ is the atomic mass of Pd. The incoherent scattering law is, for the present experimental conditions, also a fairly good description of the inelastic coherent scattering. Equation (2.5) shows that, for small energy transfers, 

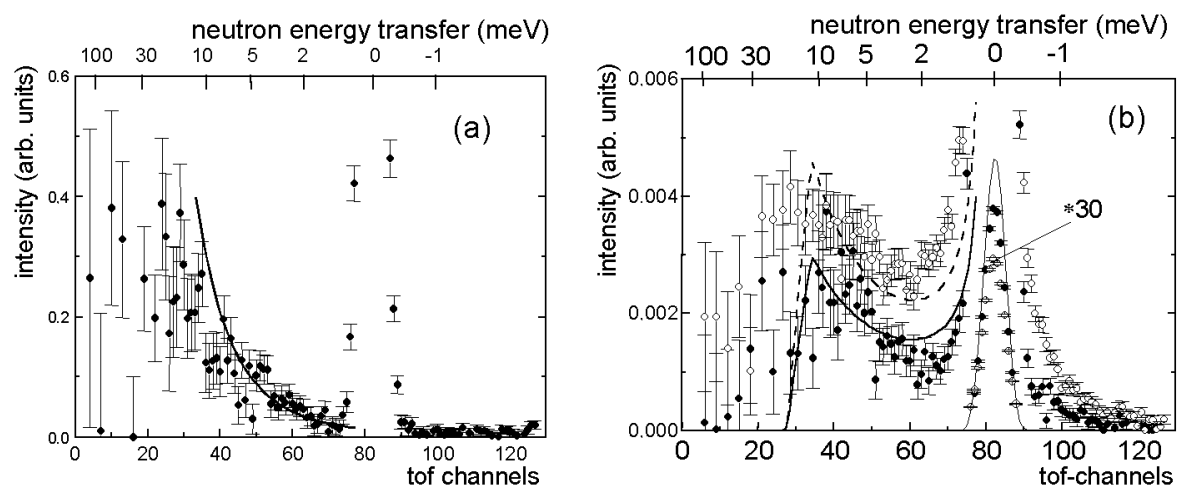

Fig. 8. Coherent (a) and incoherent (b) neutron spectra of nanocrystalline $\mathrm{PdH}_{0.03}$. The closed and open symbols represent data taken at 200 and $280 \mathrm{~K}$, respectively. The thin solid line in (b) represents the elastic line at $2 \mathrm{~K}$. The thick solid lines are the scattering functions for a VDOS $\sim \omega^{2}$ and $\operatorname{VDOS} \sim \omega$ in (a) and (b), respectively.

$\hbar \omega \rightarrow 0, S(Q, \omega)$ is very sensitive on the VDOS $f(\omega)$. In the Debye case, $f(\omega) \sim \omega^{2}$, the $S(Q, \omega)$ is constant. However, for any power law with an exponent smaller than $2, S(Q, \omega)$ increases strongly with decreasing $\omega$, and $S(Q, \omega)$ becomes zero for $\omega \rightarrow 0$ if the exponent exceeds 2 .

The scattering function for a Debye spectrum of the VDOS, $f(\omega) \sim \omega^{2}$, is indicated in Fig. 8a as thick solid line which describes the coherent data satisfactorily. On the other hand, the incoherent data in Fig. $8 \mathrm{~b}$ obviously cannot be described by this scattering law. The incoherent spectra show much higher intensities at small neutron energy transfers, compared to the coherent spectra. This shows that, at low frequencies, the local VDOS $f(\omega)$ of the Pd-atoms in the interfaces is much higher than in the lattice regions. A linear VDOS, $f(\omega)=2 \omega / \omega_{\mathrm{D}}$, where $\omega_{\mathrm{D}}=12 \mathrm{meV}$ is the cut-off frequency (thick solid lines in Fig. 8b), on the other hand, describes the incoherent data in a satisfactory manner. The unusually strong temperature dependence of the incoherent intensity at the "elastic line" is also in agreement with this VDOS. A similar behaviour was found for Pd-black [32] which indicates that the high local VDOS at low frequencies is more related to the properties of the grains than to special properties of grain boundaries. These results, a quadratic local VDOS within the grains and a linear one at the interfaces, are in very good agreement with a theoretical study of Ag-nanoclusters [36]. In a very recent molecular dynamics study of nanocrystalline $\mathrm{Ni}$ and $\mathrm{Cu}$ an enhancement of the low frequency VDOS was also found only in the regions of the grain boundaries, however this study indicates that the grain boundary VDOS is $\sim \omega^{1.5}$ rather than linear [37]. 


\section{Conclusions}

The routes followed in developing functionalized nanostructured materials exploiting unusual interfacial properties more and more rely on the knowledge of basic principles of the desired properties in relation to the nanoscale structure of the building blocks or subunits. In order to access the basic principles, characterization of structure and properties with nanometer scale or atomic resolution is essential, combined with theoretical considerations, the latter either based on phenomenological descriptions or computer simulations. The examples of magnetic nanostructures illustrates how the characterization of magnetic correlations by SANS on the nanometer scale for two quite different types of materials can give basic input for a theoretical understanding of the overall magnetic properties with the chance for dedicated developments. The molecular dynamics simulations on structure and dynamics of the interface atoms helps to understand the mechanism of defect accommodation during synthesis and plastic deformation, giving concrete directives for experimental investigations. By analyzing the vibrational modes of interface atoms these findings are complemented with the aim to verify structural details in distorted regions.

\section{References}

[1] H. Gleiter, Prog. Mater. Sci. 33, 223 (1989).

[2] J. Nanostruct. Mater. 1 (1992), Nano '94 Proc. 6 (1994), Nano '96 Proc. 9 (1997), Nano '98 Proc. in press (2000).

[3] Mater. Sci. Forum 269-272 (1998).

[4] U. Köster, U. Schünemann, in: Rapidly Solidified Alloys, Ed. H.H. Liebermann, Marcel Dekker Inc., New York 1993, p. 303.

[5] H. Van Swygenhoven, D. Farkas, A. Caro, Phys. Rev. B 62, 831 (2000).

[6] W. Wernsdorfer, E. B. Orozco, K. Hasselbach, A. Benoit, B. Barbara, N. Demoncy, A. Loiseau, H. Pascard, D. Mailly, Phys. Rev. Lett. 78, 1791 (1997).

[7] Y. Yoshizava, S. Oguma, Y. Yamauchi, J. Appl. Phys. 64, 6044 (1988).

[8] J.F. Löffler, J.P. Meier, B. Doudin, J.-P. Ansermet, W. Wagner, Phys. Rev. B 57, 2915 (1998).

[9] W. Wagner, A. Wiedenmann, W. Petry, A. Geibel, H. Gleiter, J. Mater. Res. 6, 2305 (1991).

[10] J.F. Löffler, H.B. Braun, W. Wagner, J. Appl. Phys. 85, 1 (1999).

[11] R. Harris, M. Plischke, M.J. Zuckermann, Phys. Rev. Lett. 31, 160 (1973).

[12] R. Alben, J.J. Becker, M.C. Chi, J. Appl. Phys. 49, 1653 (1978).

[13] J.F. Löffler, H.B. Braun, W. Wagner, Phys. Rev. Lett. 85, 1990 (2000).

[14] E.C. Stoner, E.P. Wohlfarth, Philos. Trans. R. Soc. Lond. A 240, 599 (1948), reprinted in IEEE Trans. Magn. 27, 3475 (1991).

[15] J.F. Löffler, Diss. ETH Zürich Nr. 12417, 1998. 
[16] Y. Yoshizawa, S. Oguma, K. Yamauchi, J. Appl. Phys. 64, 6044 (1988).

[17] G. Herzer, IEEE Trans. Magn. 25, 3327 (1989).

[18] B. Hofmann, T. Reininger, H. Kronmüller, Phys. Status Solidi A 134, 247 (1992); A. Hernando, M. Vazquez, T. Kulik, C. Prados, Phys. Rev. B 51, 3581 (1995).

[19] J. Kohlbrecher, A. Wiedenmann, H. Wollenberger, Z. Phys. B 104, 1 (1997).

[20] A. Wiedenmann, J. Appl. Crystallogr. 33, 428 (2000).

[21] A.R. Yavari, G. Fish, S.K. Das, L.A. Davis, Mater. Sci. Eng. A 182/183, 1415 (1994); A.R. Yavari, O. Drbohlav, Mat. Trans. JIM (Trans, Japan Inst. Met.) 36, 896 (1995).

[22] P. Keblinski, D. Wolf, S.R. Phillpot, H. Gleiter, Scr. Mater. 41, 631 (1999).

[23] H. Van Swygenhoven, D. Farkas, A. Caro, Phys. Rev. B 62, 831 (2000).

[24] R.A. Masumura, P.M. Hazzledine, C.S. Pande, Acta Mater. 46, 4527 (1998).

[25] H. Van Swygenhoven, M. Spaczer, A. Caro, Acta Mater. 47, 3117 (1999).

[26] H. Van Swygenhoven, M. Spaczer, D. Farkas, A. Caro, Phys. Rev. B 60, 2 (1999).

[27] H. Van Swygenhoven, M. Spaczer, A. Caro, Phys. Rev. B 58, 11246 (1998).

[28] D.J. Honneycutt, H.C. Andersen, J. Phys. Chem. 91, 4950 (1987).

[29] T. Ungar, S. Ott, P.G. Sanders, A. Borbély, J.R. Weertman, Acta Mater. 46, 3693 (1998); T. Ungar, A. Revesz, A. Borbely, J. Appl. Crystallogr. 31, 554 (1998).

[30] B. Fultz, J.L. Robertson, T.A. Stephens, L.J. Nagel, S. Spooner, J. Appl. Phys. 79, 8318 (1996).

[31] B. Fultz, C.C. Ahn, E.E. Alp, W. Sturhahn, T.S. Toellner, Phys. Rev. Lett. 79, 937 (1997).

[32] U. Stuhr, H. Wipf, K.H. Andersen, H. Hahn, Phys. Rev. Lett. 81, 1449 (1998).

[33] U. Stuhr, H. Wipf, T. J. Udovic, J. Weißmüller, H. Gleiter, J. Phys., Condens. Matter 7, 219 (1995).

[34] T. Striffler, U. Stuhr, H. Wipf, H. Hahn, S. Egelhaaf, Eur. Phys. J. B 17, 245 (2000).

[35] H.R. Schober, V. Lottner, Z. Phys. Chem. 114, 351 (1979).

[36] A. Kara, T.S. Rahman, Phys. Rev. Lett. 81, 1453 (1998).

[37] P.M. Derlet, R. Meyer, L.J. Lewis, U. Stuhr, H. Van Swygenhoven, Phys. Rev. Lett. 87, 205501 (2001). 